\title{
Selección de hongos antagonistas para el control biológico de Botrytis cinerea en viveros forestales en Chile
}

\author{
Screening to antagonistic fungi for Botrytis cinerea \\ biocontrol in Chilean forest nurseries
}

\author{
Gloria Molina Mercader ${ }^{1}$, Salomé Zaldúa Flores², Gastón González Vargas'1, \\ Eugenio Sanfuentes Von Stowasser ${ }^{2 *}$ \\ ${ }^{1}$ Biocaf Ltda., Camino a Coronel, Concepción. \\ *Autor de correspondencia: ${ }^{2}$ Universidad de Concepción, Facultad de Ciencias Forestales, \\ Laboratorio de Patología Forestal, Victoria 631, Concepción, esanfuen@udec.cl
}

\begin{abstract}
SUMMARY
Botrytis cinerea is one of the most important pathogens in Chilean forest nurseries. The disease control has been based on the use of fungicides; nevertheless, under certain conditions of disease pressure this practice has not been effective, besides its environmental problems due to its excessive use. The objective of this study was to select antagonistic fungi to $B$. cinerea, by means in vitro and nurseries assays, to determine its capacity as biocontrol agents of the «grey mould» disease in forest nurseries. The antagonistic potentials were obtained from the plants phyloplane, collected from forest nurseries. Seventy one fungi strains were evaluated in their capacity to reduce in vitro the pathogen colonization and sporulation by bioassays in Eucalyptus leaf discs. Selected strains were assayed under greenhouse conditions. Pinus radiata and E. globulus plants were sprayed with the pathogen $\left(1 \times 10^{5}\right.$ conidias $\left./ \mathrm{ml}\right)$ and later treated with the antagonists $\left(1 \times 10^{7}\right.$ conidias $\left./ \mathrm{ml}\right)$, evaluating the disease incidence and severity. In the in vitro assays, four strains of Trichoderma, three of Clonostachys, four of Penicillium, one of Cladosporium and other eight unidentified fungi strains did not significantly reduce pathogen colonization and sporulation. In the greenhouses assays, the strain Clonostachys (A-10) was able to reduce, in both $P$. radiata and E. globules, the disease incidence and severity. These results allow concluding that antagonists selected have the potential to $B$. cinerea control in forest nurseries.
\end{abstract}

Key words: biological control, Clonostachys, Trichoderma, forest nurseries diseases, Botrytis cinerea.

\section{RESUMEN}

Botrytis cinerea es uno de los patógenos más importante en viveros forestales en Chile. El control de la enfermedad se ha basado en el uso de fungicidas; sin embargo, bajo ciertas condiciones de presión de enfermedad esta medida no ha sido eficaz, sumado a problemas ambientales debido a su uso excesivo. El objetivo de este estudio fue seleccionar hongos antagonistas a $B$. cinerea, mediante ensayos in vitro y de invernadero, y determinar su capacidad como agentes biocontroladores de este hongo en viveros forestales. Los potenciales antagonistas fueron obtenidos a partir del filoplano de plantas, colectadas desde viveros forestales. Fueron ensayadas 71 cepas de hongos, evaluadas en su capacidad para reducir la colonización y esporulación del patógeno en ensayos in vitro, mediante bioensayos en discos de hojas de Eucalyptus globulus. Las cepas seleccionadas fueron ensayadas bajo condiciones de invernadero. Las plantas de Pinus radiata y E. globulus fueron pulverizadas con el patógeno (1x $10^{5}$ conidias/ml) y después tratadas con los antagonistas ( 1 x $10^{7}$ conidias/ml), evaluándose la incidencia y severidad de la enfermedad. En los ensayos in vitro, cuatro cepas de Trichoderma, tres de Clonostachys, cuatro de Pencillium, una de Cladosporium y otras ocho cepas de hongos no identificados redujeron significativamente la colonización y esporulación del patógeno. En los ensayos en invernadero, la cepa Clonostachys (A-10) fue capaz de reducir, tanto en P. radiata como en E. globulus la incidencia y severidad de la enfermedad. Estos resultados permiten concluir el potencial de los antagonistas seleccionados en el control de B. cinerea.

Palabras clave: control biológico, Clonostachys, Trichoderma, enfermedades en viveros forestales, Botrytis cinerea.

\section{INTRODUCCIÓN}

La enfermedad "moho gris", causada por Botrytis cinerea Pers. ex Fr., se presenta en diversos cultivos, incluyendo viveros forestales de la V a la X Regiones de
Chile, donde afecta, preferentemente, a plantas del género Eucalyptus. Este patógeno también ha sido descrito sobre plántulas de Pinus radiata D. Don, durante su viverización, atacando la porción apical (Butin y Peredo 1986). Tanto en $P$. radiata como en especies de Eucalyp- 
tus, B. cinerea constituye un serio problema de pudrición en estaca durante las primeras etapas del establecimiento o "estaquillado".

Los factores que favorecen la infección por B. cinerea son aquellos que resultan en daño físico producido a la planta (Coley-Smith et al. 1980, Figueredo et al. 2001, Sanfuentes y Ferreira 1997), que en el caso particular en viveros de $P$. radiata y Eucalyptus spp. en Chile, es especialmente importante el daño causado por heladas.

Una vez introducido $B$. cinerea en los cultivos, puede sobrevivir en sustrato orgánico, hojas muertas caídas en la superficie de los recipientes y en tejidos de plántulas como componente de la biota del filoplano (Sanfuentes y Ferreira 1997, Sharabani et al. 1999, Pande et al. 2001, Barnes y Shaw 2003).

Tradicionalmente el principal método de control de $B$. cinerea ha sido mediante el uso de fungicidas. Aunque estos continúan siendo el soporte del manejo del "moho gris", recientes estudios han constatado una menor efectividad en las aplicaciones, producto del abrupto aumento de los niveles de resistencia del patógeno a fungicidas benzimidazoles, como también un desarrollo paulatino de resistencia a dicarboximidas, por el uso repetitivo de estos grupos de fungicidas (Ferreira 1989, Zhang et al. 1994, Col et al. 1994, Esterio y Auger 1997).

El público evidencia un descontento con residuos de fungicidas en el follaje y frutos; asimismo, se percibe una animadversión de la población contra la aplicación de productos químicos que dañan al hombre y al ambiente. La tendencia gubernamental a legislar restricciones en el uso de plaguicidas ha incentivado investigaciones orientadas a proporcionar nuevas alternativas de control efectivos y que disminuyan los residuos de agrotóxicos, incorporando medidas de manejo cultural y biológico (Zhang et al. 1994, Elad y Zimand 1991).

En este contexto, aparece como una alternativa viable la aplicación de un método biológico, especialmente diseñado como una estrategia dentro del concepto de manejo integrado de enfermedades.

Numerosos estudios realizados en cultivos de albahaca, garbanzo, frutilla, frambuesa, uva de mesa y vinífera, cebolla, tomate, bulbos de flores, especies de eucaliptos y píceas, han mostrado la efectividad del control biológico sobre $B$. cinerea, y que en algunos casos ha sido superior que los fungicidas recomendados para el cultivo (Sanfuentes y Ferreira 1997, Zhang et al. 1994, Esterio y Auger 1997, Peng y Sutton 1991, Elad et al. 1994, Zhang et al. 1996, Yu y Sutton 1997, 1999, Swadling y Jeffries 1995, Cole et al. 2004, Chaves y Wang 2004).

En Chile, el control biológico de B. cinerea, mediante antagonistas específicos, ha sido poco explorado. Entre los casos operacionales que es posible citar, se encuentran la satisfactoria protección de vides, mediante el producto Trichodex ${ }^{\circledR}$, elaborado a base del hongo Trichoderma harzianum $\left(\mathrm{T}_{39}\right)$ (Esterio y Auger 1997). El uso de este tipo de "botriticidas" aún no se ha aplicado en viveros forestales, donde $B$. cinerea es el principal problema biótico en la producción de plantas de E. globulus en contenedores.

Debido a los problemas mencionados previamente, y a la evidencia de éxito obtenido en otros cultivos en la aplicación de control biológico, esta investigación se planteó como objetivo principal seleccionar antagonistas contra $B$. cinerea, para ser empleados en el control biológico del "moho gris" en viveros forestales.

\section{MÉTODOS}

Aislamiento de B. cinerea y producción de inóculo. La cepa de $B$. cinerea utilizada en los ensayos fue aislada a partir de plantas de $P$. radiata, con síntomas de "moho gris", provenientes del Vivero Carlos Douglas, propiedad de Forestal Mininco S.A. localizado en la comuna de Yumbel, VIII Región.

Acículas y tallos de las plantas fueron seccionados en trozos y dispuestos dentro de placas Petri, con papel absorbente humedecido en agua destilada estéril (ADE), conformando una cámara húmeda para estimular la esporulación del patógeno.

Una vez producida la esporulación, se colectaron las conidias mediante estilete estéril, las que fueron sembradas en placas Petri conteniendo agar papa dextrosa (APD), con $100 \mu \mathrm{g} / \mathrm{mL}$ de sulfato de estreptomicina (SE) y repicadas hasta obtener cultivos puros. Luego, estos fueron almacenados en tubos de ensayo con APD inclinado y mantenidos a $4^{\circ} \mathrm{C}$ hasta su uso.

Para la producción de inóculo, se procedió a repicar desde la cepa almacenada a tubos con medio de cultivo APD e incubados a $22 \pm 1^{\circ} \mathrm{C}$, con fotoperiodo de $12 \mathrm{~h}$, durante diez días. Las esporas producidas en los cultivos se colectaron con ADE, para luego determinar la concentración de conidias en la suspensión mediante hematocitómetro, previo a la aplicación.

Aislamiento y producción de inóculo de antagonistas. Se realizaron visitas a viveros forestales de la VII y VIII Región, entre los meses de mayo a junio de 2001, recolectándose muestras de plantas de E. globulus y $P$. radiata. Las plantas seleccionadas no presentaban síntomas o signos de enfermedades.

Desde el follaje de estas plantas se procedió a realizar los aislamientos para obtener las cepas de hongos potenciales antagonistas a $B$. cinerea, basándose en la metodología descrita por Peng y Sutton (1991). En este método, se utilizaron $30 \mathrm{~g}$ de hojas de E. globulus y de acículas de $P$. radiata, que fueron seccionadas y colocadas, por separado, en matraces de Erlenmeyer con $250 \mathrm{ml}$ de ADE y $50 \mu \mathrm{L} / \mathrm{L}$ de Tween 20 y mantenida en suspensión, en agitación continua durante $30 \mathrm{~min}$ (Sanfuentes y Ferreira 1997, Peng y Sutton 1991). Desde la suspensión en agitación se tomaron alícuotas de $1 \mathrm{~mL}$, procediéndose a 
realizar diluciones hasta $10^{-2}$. De la última, se depositaron alícuotas de $0,5 \mathrm{ml}$, en placas Petri que contenían APD con $100 \mathrm{mg} / \mathrm{L}$ de SE, e incubadas durante cinco días a $22 \pm 1^{\circ} \mathrm{C}$, en oscuridad (Sanfuentes y Ferreira 1997).

Las colonias de hongos obtenidas se repicaron a tubos con APD inclinado, los que fueron almacenados a $4 \pm 1^{\circ} \mathrm{C}$ hasta su utilización en los ensayos de selección.

Para la producción de inóculo, las cepas almacenadas fueron repicadas a placas Petri conteniendo APD e incubadas a $25 \pm 1^{\circ} \mathrm{C}$ durante cinco días. Las esporas producidas en los cultivos se colectaron con ADE, determinándose la concentración de conidias en la suspensión mediante un hematocitómetro.

Selección de antagonistas. La selección de antagonistas fue realizada, en una primera etapa, utilizando ensayos in vitro de acuerdo a la metodología descrita por Peng y Sutton (1991) adaptada por Sanfuentes y Ferreira (1997). Discos de hojas de E. globulus tratados previamente con hipoclorito de sodio comercial (10\%) fueron dispuestos en placas Petri estableciendo una cámara húmeda.

Sobre los discos fueron aplicados los hongos potenciales antagonistas en una concentración de $10^{7}$ conidias $/ \mathrm{mL}$ y luego de 24 horas se asperjó $B$. cinerea en concentración de $10^{5}$ conidias $/ \mathrm{mL}$, adicionada con glucosa $1 \mathrm{~g} / \mathrm{L}$. Al día siguiente, los discos de hojas fueron colocados en medio de cultivo PCA, que contenía agar $(20 \mathrm{~g} / \mathrm{L})$, paraquat (dicloruro de paraquat) $1 \mathrm{~g} / \mathrm{L}$ y cloranfenicol $50 \mathrm{mg} / \mathrm{L}$, siendo incubados durante siete días a $26 \pm 1^{\circ} \mathrm{C}$.

Fueron probadas 71 cepas de hongos, en siete ensayos, debido a la dificultad de evaluarlas simultáneamente. Cada cepa fue evaluada empleando cuatro repeticiones, y la unidad experimental fue la placa Petri que contenía 10 discos de hojas. El tratamiento testigo sólo recibió la aplicación de $B$. cinerea. Adicionalmente, en cada ensayo se incluyeron dos unidades experimentales, sin tratamiento alguno, para observar la presencia de microorganismos endófitos.

Para la evaluación del ensayo se confeccionó una escala visual de acuerdo a Peng y Sutton (1991), obteniéndose un Índice de Esporulación por Tratamiento (IET), que consideró la colonización y abundancia de conidióforos de B. cinerea en la superficie del disco de hoja de E. globulus (cuadro 1)

El cálculo de este índice de esporulación por tratamiento se realizó a través de la siguiente fórmula:

$$
I E T=\frac{\sum_{j=1}^{N} G E_{j} \times n_{j}}{N}
$$

Donde IET correspondió al índice de esporulación por tratamiento, $G E_{j}$ fue el grado de esporulación asignado según escala visual, $n_{j}$ la cantidad de discos en el grado y $N$ el número total de discos evaluados.
Efecto de cepas seleccionadas en la supresión de B. cinerea, aplicados antes y después del patógeno. Las cepas de Trichoderma A6, A27, A30 y A58 y Clonostachys A10, A11 A14 y A32, que presentaron los mayores niveles de inhibición de $B$. cinerea en la selección in vitro, fueron ensayadas en dos ocasiones. El primer ensayo fue de acuerdo a la metodología descrita previamente en el ensayo de selección, y en el segundo ensayo sólo fue modificada la aplicación de $B$. cinerea, que se realizó 24 h después de la cepa de antagonista.

Efecto de los antagonistas en el control de B. cinerea en ensayos sobre E. globulus en vivero. El ensayo fue conducido en el invernadero de la empresa Biocaf, ubicado en el predio Escuadrón, camino a Coronel, VIII Región. Fueron utilizadas plantas de E. globulus proporcionadas por Forestal Mininco S.A., las que presentaban buen estado sanitario, de $30 \mathrm{~cm}$. de altura y diámetro a la altura del cuello (DAC) de $0,5 \mathrm{~cm}$.

Los tratamientos aplicados fueron las cepas de Trichoderma (A6, A27, A30 y A58) y Clonostachys (A10, A11, A14 y A32), el fungicida fehexamid (Teldor 50\% WP) en dosis $1 \mathrm{~g} / \mathrm{L}$ y el control, en que sólo fue aplicado B. cinerea. El inóculo de los antagonistas y patógeno fueron aplicados en concentraciones de $10^{7}$ y $10^{5}$ conidias/ml en ADE, respectivamente. En la suspensión de esporas de $B$. cinerea, se adicionó glucosa (1 g/L) (Elad et al. 1994).

El patógeno fue aplicado a la totalidad de las plantas el 15 de septiembre del 2003 y al día siguiente se aplicaron las suspensiones de esporas de los antagonistas seleccionados. Ambas aplicaciones de patógeno y antagonistas se repitieron quince días después en la misma secuencia. El ensayo en su totalidad duró dos meses.

El riego por aspersión fue suspendido por 48 h desde el día de aplicación del patógeno, regándose sólo al cuello de la planta, para evitar mojar el follaje. Se registraron diariamente temperatura y humedad tres veces al día.

Cuadro 1. Escala visual basada en el grado de colonización y esporulación de $B$. cinerea (GE) en discos de hojas de $E$. globulus.

Visual scale based on B. cinerea colonization and sporulation level (GE) in E. globulus leaf disks assays

\begin{tabular}{cl}
\hline GE & Descripción \\
\hline 1 & Sin presencia de B. cinerea. \\
2 & Esporulación menor del $25 \%$. \\
3 & Esporulación entre 25 y $50 \%$. \\
4 & Esporulación mayor del $50 \%$. \\
5 & Esporulación 100\%, conidióforos dispersos. \\
6 & Esporulación 100\%, conidióforos se encuentran \\
& distribuidos en forma irregular. \\
7 & Conidióforos se observan colonizando todo el \\
& disco en forma abundante y homogénea.
\end{tabular}


Cada tratamiento consistió de cuatro repeticiones, y la unidad experimental estuvo constituida por 80 plantas (contenidas en una bandeja), las que fueron distribuidas totalmente al azar dentro del invernadero.

Para la evaluación se colectaron seis plantas por bandeja, 24 plantas por tratamiento, a las cuales se les cortó la raíz para luego ser colocadas en bolsas transparentes conteniendo algodón estéril embebido en ADE, conformando una cámara húmeda que fue mantenida a $22 \pm 1^{\circ} \mathrm{C}$, durante siete días. Posteriormente, las plantas fueron revisadas con lupa estereoscópica (x 8), determinando la presencia de signos del patógeno, en el tallo y follaje, de acuerdo a la siguiente escala elaborada para tal efecto: (1) ausencia de esporulación, (2) esporulación < 12\%, (3) esporulación $>25 \%$ y $<50 \%$, (4) esporulación $>50 \%$ y (5) $100 \%$ de esporulación. En cada tratamiento se obtuvo el índice de esporulación aplicando la fórmula IET [1], donde $G E_{j}$ fue el grado de severidad asignado, según la escala visual, $n_{j}$ la cantidad de plantas en el grado y $N$ el número total de plantas evaluadas por bandeja.

Se calcularon la incidencia promedio $(I P)$ del patógeno y el porcentaje utilizando la siguiente fórmula:

$$
I P=\frac{\sum_{j=1}^{j} \frac{n_{j}}{N_{j}}}{R}
$$

Donde $n_{j}$ corresponde a la cantidad de plantas con presencia de $B$. cinerea en la repetición, $N_{j}$ cantidad total de plantas en la repetición y $R$ cantidad de repeticiones por tratamiento.

Los porcentajes de control para cada tratamiento se calcularon de la siguiente forma:

Porcentaje de control $=100-\left(\frac{\mathrm{IP} \text { antagonista } \cdot 100}{\mathrm{IP} \text { control }}\right)$

Efecto de los antagonistas en el control de B. cinerea en ensayos sobre $P$. radiata en vivero. El ensayo se realizó en el invernadero del vivero "Carlos Douglas", de propiedad de la empresa Forestal Mininco S.A., localizado en la Comuna de Yumbel, VIII Región. Fueron empleadas plantas de $P$. radiata de 8 meses de edad, de aproximadamente $40 \mathrm{~cm}$ de altura y $1 \mathrm{~cm}$ de DAC, que presentaban buen estado sanitario.

Los antagonistas fueron asperjados sobre el follaje de las plantas el 15 de mayo de 2004, a una concentración de $10^{7}$ conidios $/ \mathrm{mL}$, en tres oportunidades, a intervalos de 15 días. Adicionalmente, se realizaron dos aplicaciones $48 \mathrm{~h}$ antes que los antagonistas, con $B$. cinerea a $10^{5}$ conidios/mL, coincidiendo con la primera y segunda aplicaciones de los antagonistas, con la finalidad de incrementar y homogeneizar la presencia de inóculo del pató- geno. Los fungicidas fueron aplicados en las mismas ocasiones que los antagonistas. El ensayo tuvo una duración total de 45 días.

Después de la primera aplicación del patógeno y los antagonistas, en cada oportunidad las plantas se mantuvieron cubiertas bajo plástico transparente durante $24 \mathrm{~h}$, para asegurar el mojamiento foliar y favorecer la germinación de las esporas del patógeno y antagonistas. Posteriormente, se mantuvo alta humedad relativa, mediante nebulización periódica. Se registró diariamente la humedad relativa y la temperatura tres veces al día $(9,12$ y $17 \mathrm{~h})$.

El ensayo consistió de seis tratamientos; dos cepas de Trichoderma (A6 y A27), dos de Clonostachys (A10 y A11), el tratamiento fungicida, mediante la aplicación intercalada de Switch (cyprodinil), Sumisclex (fludioxonil + procymidona), en dosis de $3,3 \mathrm{~g} / \mathrm{L}$ y el tratamiento control, que sólo recibió aplicación del patógeno. Fueron empleadas cuatro repeticiones por tratamiento y la unidad experimental estuvo constituida por 96 plantas (bandeja), las que fueron distribuidas totalmente al azar dentro del invernadero.

Se evaluaron incidencia y severidad de la enfermedad en las 60 plantas centrales de la cada bandeja. Para la severidad cada planta se dividió en tres tercios, donde se observó la presencia o ausencia de signos del patógeno, de acuerdo a escala descrita previamente.

Análisis estadístico. Todos los ensayos siguieron un diseño experimental completamente al azar. El análisis de los resultados (IET) se realizó mediante la prueba no paramétrica de Kruskal-Wallis con el auxilio del programa STATISTICA. Para las comparaciones múltiples se utilizó la prueba de contraste de Kruskal-Wallis con $95 \%$ de confianza.

\section{RESULTADOS}

Ensayos in vitro. Selección de antagonistas. De entre 144 cepas de hongos aisladas desde el filoplano de plantas de $P$. radiata y $E$. globulus, fueron evaluadas 71 cepas, privilegiando para las pruebas de selección aquellas pertenecientes al género Trichoderma y de la especie Clonostachys rosea (= Gliocladium roseum) (cuadro 2). De las cepas ensayadas, 20 (28, 2\% del total) redujeron significativamente la colonización y esporulación de B. cinerea, de las cuales el $60 \%$ presentó IET menor a 2. Entre las cepas que evidenciaron mayor efecto inhibitorios sobre la esporulación de B. cinerea, tres pertenecieron a la especie C. rosea (A10, A11, A32) con IET entre 1,0 y 1,37, y otras cuatro al género Trichoderma (A6, A27, A30 y A58) con IET entre 1,0 y 1,9 (cuadro 2). 
Cuadro 2. Esporulación de B. cinerea (IET) en discos de hojas de E. globulus tratados con 71 cepas de hongos antagonistas. Botrytis cinerea colonization and sporulation (IET) in E. globulus leaf disks assays treated with antagonists fungi strains.

\begin{tabular}{|c|c|c|c|c|c|c|c|c|c|c|c|c|c|c|c|c|c|c|c|}
\hline \multicolumn{20}{|c|}{ Ensayos } \\
\hline \multicolumn{3}{|c|}{1} & \multicolumn{3}{|c|}{2} & \multicolumn{2}{|c|}{3} & \multicolumn{3}{|c|}{4} & \multicolumn{3}{|c|}{5} & \multicolumn{3}{|c|}{6} & \multicolumn{3}{|c|}{7} \\
\hline Cepa & IET & & Cepa & IE & & Сера & IET & Сepa & IE & & Cepa & & $\mathrm{T}$ & Cepa & IET & & Cepa & IE & $T$ \\
\hline $\mathrm{A} 1$ & 1,65 & & A6 & 1,00 & $\mathrm{a}$ & A18 & 5,32 & A23 & 4,80 & $\mathrm{bc}$ & A31 & 4,25 & bcdef & A45 & 3,03 & $a b c$ & A58 & & $\mathrm{a}$ \\
\hline A2 & 4,22 & $a b$ & A7 & 1,03 & $a b$ & A21 & 5,80 & A24 & 6,30 & $\mathrm{c}$ & A32 & 1,37 & $\mathrm{a}$ & A46 & 1,00 & $\mathrm{a}$ & A59 & 4,4 & $a b c$ \\
\hline A3 & 6,72 & & A9 & 6,83 & $\mathrm{c}$ & A 22 & 6,33 & A25 & 4,53 & $\mathrm{bc}$ & A33 & 2,35 & $a b c$ & $\mathrm{~A} 47$ & 6,22 & $\mathrm{~d}$ & A60 & 6,6 & de \\
\hline A4 & 6,65 & & $\mathrm{~A} 10$ & 1,00 & $\mathrm{a}$ & $\mathrm{C}$ & 6,88 & A26 & 3,03 & $\mathrm{bc}$ & A34 & 4,17 & cdef & A48 & 4,92 & $\mathrm{~cd}$ & A61 & 5,7 & $\mathrm{~cd}$ \\
\hline A5 & 1,57 & & A11 & 1,10 & $a b$ & - & - & A27 & 1,37 & $\mathrm{a}$ & A35 & 5,62 & $\mathrm{f}$ & A49 & 3,20 & bcd & A62 & 6,4 & de \\
\hline C** & 4,73 & & A12 & 1,57 & $b c$ & - & - & A28 & 1,37 & $\mathrm{a}$ & A36 & 4,75 & ef & A50 & 2,22 & $a b c$ & A63 & 7,0 & $\mathrm{e}$ \\
\hline- & - & & A13 & 1,57 & $\mathrm{bc}$ & - & - & A29 & 1,98 & $a b$ & A37 & 3,90 & bcdef & A51 & 1,40 & $\mathrm{ab}$ & A64 & 5,3 & bcd \\
\hline- & - & & A14 & 1,57 & $\mathrm{bc}$ & - & - & A 30 & 1,45 & $\mathrm{a}$ & A38 & 2,47 & abcd & A52 & 1,00 & $\mathrm{a}$ & A65 & 6,3 & de \\
\hline- & - & & A15 & 1,57 & $\mathrm{bc}$ & - & - & $\mathrm{C}$ & 5,3 & $\mathrm{bc}$ & A39 & 2,90 & abcde & A53 & 1,00 & $\mathrm{a}$ & A66 & 5,9 & cde \\
\hline- & - & & $\mathrm{C}$ & 4,73 & $\mathrm{c}$ & - & - & - & - & & A40 & 4,58 & def & A54 & 5,32 & $\mathrm{~cd}$ & A67 & 2,9 & $a b$ \\
\hline - & - & & - & - & & - & - & - & - & & A41 & 2,32 & $a b$ & A55 & 4,92 & $\mathrm{~cd}$ & A68 & 5,5 & bcd \\
\hline- & - & & - & - & & - & - & - & - & & A42 & 2,93 & abcde & A56 & 5,10 & $\mathrm{~cd}$ & A69 & 5,9 & cde \\
\hline- & - & & - & - & & - & - & - & - & & A43 & 4,80 & ef & A57 & 3,10 & bcd & A70 & 5,5 & bcd \\
\hline- & - & & - & - & & - & - & - & - & & A44 & 5,53 & $\mathrm{f}$ & $\mathrm{C}$ & 3,3 & bcd & A71 & 4,6 & $a b c$ \\
\hline- & - & & - & - & & - & - & - & - & & $\mathrm{C}$ & 6,3 & $\mathrm{f}$ & - & - & & $\mathrm{C}$ & 6,7 & de \\
\hline
\end{tabular}

* Índice de esporulación por tratamiento.

** Control, sólo aplicación de B. cinerea

Letras diferentes en la columna indican diferencias significativas $(P<0,05)$.

También se constató que otros tipos de hongos como Cladosporium sp. (A28) y Penicillium spp. (A7, A46, A52, A53) presentaron altos niveles de inhibición del patógeno, con IET entre 1,0 y 1,03 . De las restantes cepas que presentaron efecto significativo en la supresión de B. cinerea (A33, A38, A39, A41, A42, A59, A67 y A71) correspondieron a hongos demateáceos y coelomicetes no identificados, todas con IET mayor a 2,32. Tanto las cepas de Cladosporium, Penicillium, como aquellas no identificadas, fueron descartadas de los ensayos de supresión posteriores, por razones que se señalan en la discusión.

Efecto de los antagonistas seleccionados en la supresión de B. cinerea, aplicados antes y después del patógeno. Todas las cepas de antagonistas redujeron significativamente la esporulación de $B$. cinerea, presentando resultados semejantes a los obtenidos en el ensayo de selección in vitro (cuadro 3 ).

Tanto cuando los antagonistas se aplicaron en forma previa al patógeno como posterior, se obtuvo reducción de la esporulación de $B$. cinerea.

En el primer caso, la esporulación de $B$. cinerea (IET) fue cercana a 1 para los tratamientos con las cepas antagonistas A6, A10, A11 y A32 (IET 1,12 a 1,19), indicando que prácticamente se inhibió casi por completo la colonización y esporulación del patógeno en los discos de hojas. En relación a los resultados obteni-
Cuadro 3. Efecto de los antagonistas seleccionados en la supresión de B. cinerea sobre en discos de hojas de E. globulus, aplicados antes y después del patógeno.

Antagonist and fungicide effect in B. cinerea suppression on E. globulus leaf disks assays.

\begin{tabular}{lcccc}
\hline \multirow{2}{*}{ Tratamiento } & \multicolumn{4}{c}{ Aplicación antagonista* } \\
\cline { 2 - 5 } & \multicolumn{2}{c}{ Antes } & \multicolumn{2}{c}{ Después } \\
& IET & Rango & IET & Rango \\
\hline Trichoderma (A6) & $1,13 \mathrm{a}$ & $1,0-1,5$ & $1,98 \mathrm{~b}$ & $1,8-2,2$ \\
Clonostachys (A10) & $1,13 \mathrm{a}$ & $1,0-1,5$ & $1,20 \mathrm{a}$ & $1,0-1,6$ \\
Clonostachys (A11) & $1,12 \mathrm{a}$ & $1,0-1,3$ & $1,17 \mathrm{a}$ & $1,0-1,5$ \\
Clonostachys (A14) & $1,37 \mathrm{a}$ & $1,2-1,6$ & $1,20 \mathrm{a}$ & $1,0-1,6$ \\
Trichoderma (A27) & $1,38 \mathrm{a}$ & $1,2-1,6$ & $1,25 \mathrm{a}$ & $1,0-1,5$ \\
Trichoderma (A30) & $1,41 \mathrm{a}$ & $1,1-1,7$ & $1,88 \mathrm{~b}$ & $1,8-2,0$ \\
Clonostachys (A32) & $1,19 \mathrm{a}$ & $1,0-1,7$ & $1,05 \mathrm{a}$ & $1,0-1,1$ \\
Trichoderma (A58) & $1,73 \mathrm{~b}$ & $1,1-2,4$ & $1,90 \mathrm{~b}$ & $1,8-2,0$ \\
B. cinerea & $6,35 \mathrm{c}$ & $5,7-7,0$ & $4,6 \mathrm{c}$ & $4,5-4,8$ \\
\hline
\end{tabular}

* Letras diferentes en la columna indican diferencias significativas $(P<0,05)$.

dos en el ensayo de selección, las cepas A6, A10, A11 y A27 presentaron una leve disminución en la eficiencia en la supresión de la esporulación, contrariamente las cepas A14, A30, A32 y A58 aumentaron la eficiencia en control. 
En el segundo caso, todos los antagonistas fueron capaces de reducir la esporulación del patógeno, presentando diferencias significativas con el tratamiento control (cuadro 3). Al igual que en el caso anterior, se verificó una disminución en la efectividad de algunas cepas en el control de $B$. cinerea, con respecto al ensayo de selección, en cambio las cepas de Clonostachys A14 y A32 y Trichoderma A27 aumentaron levemente el nivel de control del patógeno.

Efecto de los antagonistas en el control de B. cinerea en ensayos sobre E. globulus en vivero. En el ensayo de antagonistas sobre E. globulus todas las cepas de hongos antagonistas fueron capaces de controlar $B$. cinerea (cuadro 4), presentando diferencias significativas con el control. Los mejores resultados se obtuvieron con las cepas Clonostachys A10, A11 y Trichoderma A27, todas con IET $\leq 1,3$ y reducción en la incidencia mayor o igual a $85 \%$, verificándose el potencial de estos antagonistas para el control de $B$. cinerea, y superando al tratamiento con fungicida, el cual no tuvo efecto sobre el patógeno (cuadro 4).

Se observó esporulación sobre el tallo de las plantas tanto de los antagonistas como del patógeno, y en hojas sólo se constató esporulación de los antagonistas. Además, se detectó ocasionalmente la presencia en las hojas de otros hongos, como Pestalotiopsis spp. y Penicillium spp.

Estos resultados son comparables con los obtenidos en los ensayos in vitro, referentes a la efectividad de los antagonistas en la supresión de la esporulación de B. cinerea. En este ensayo, los antagonistas A6, A10, A11, A14, A27, A30 y A32 fueron eficientes en el con-

Cuadro 4. Efecto de los antagonistas y fungicidas sobre B. cinerea, en plantas de E. globulus, en ensayo de invernadero Biocaf.

Antagonist and fungicide effect against $B$. cinerea on E. globulus plants, Biocaf nursery assay.

\begin{tabular}{lccc}
\hline \multicolumn{1}{c}{ Tratamiento } & IET & Rango & $\begin{array}{c}\text { IP } \\
\text { B. cinerea }(\%)^{* * *}\end{array}$ \\
\hline Trichoderma (A6) & $1,7 \mathrm{a}^{*}$ & $1,3-2,2$ & $20,8(75)$ \\
Clonostachys (A10) & $1,3 \mathrm{a}$ & $1,0-1,7$ & $12,5(85)$ \\
Clonostachys (A11) & $1,3 \mathrm{a}$ & $1,0-1,7$ & $8,3(90)$ \\
Clonostachys (A14) & $1,5 \mathrm{a}$ & $1,3-2,0$ & $16,7(80)$ \\
Trichoderma (A27) & $1,2 \mathrm{a}$ & $1,0-1,7$ & $8,3(90)$ \\
Trichoderma (A30) & $1,5 \mathrm{a}$ & $1,2-2,0$ & $20,8(75)$ \\
Clonostachys (A32) & $1,4 \mathrm{a}$ & $1,3-1,7$ & $12,5(85)$ \\
Trichoderma (A58) & $2,0 \mathrm{~b}$ & $1,7-2,5$ & $45,8(45)$ \\
Teldor 50\% WP & $4,7 \mathrm{c}$ & $4,5-4,8$ & $75,0(10)$ \\
B. cinerea $(* *)$ & $4,8 \mathrm{c}$ & $4,8-4,8$ & 83,3 \\
\hline
\end{tabular}

* Letras diferentes en la columna indican diferencias significativas $(P<0,05)$

** Sólo aplicación de $B$. cinerea (control).

*** Porcentaje de control en relación al tratamiento con $B$. cinerea
Cuadro 5. Efecto de antagonistas y fungicidas sobre $B$. cinerea en plantas de $P$. radiata, ensayo vivero Carlos Douglas.

Antagonist and fungicide effect against $B$. cinerea in $P$. radiata plants, Carlos Douglas nursery assay.

\begin{tabular}{llcl}
\hline \multicolumn{1}{c}{ Tratamientos } & IET & Rango & $\begin{array}{c}\text { IP } \\
\text { B. cinerea }(\%)^{* * *}\end{array}$ \\
\hline Switch-Sumisclex & $1,07 \mathrm{a} *$ & $1,06-1,07$ & $15,8(77,8)$ \\
Clonostachys (A10) & $1,21 \mathrm{~b}$ & $1,05-1,30$ & $42,6(40,3)$ \\
Clonostachys (A11) & $1,29 \mathrm{bc}$ & $1,19-1,36$ & $55,4(22,3)$ \\
Trichoderma (A27) & $1,37 \mathrm{c}$ & $1,24-1,54$ & $64,6(9,4)$ \\
Trichoderma (A6) & $1,43 \mathrm{c}$ & $1,34-1,50$ & $71,9(-0,8)$ \\
B. cinerea $(* *)$ & $1,37 \mathrm{c}$ & $1,28-1,51$ & 71,3 \\
\hline
\end{tabular}

* Letras diferentes en la columna indican diferencias significativas $(P<0,05)$.

** Sólo aplicación de $B$. cinerea.

*** Porcentaje de control en relación al tratamiento con $B$. cinerea.

trol del patógeno, presentando IET inferior a 1,7 y una reducción de la incidencia entre 75 y $90 \%$.

Efecto de los antagonistas en el control de B. cinerea en ensayos sobre $P$. radiata en vivero. En el ensayo de antagonistas sobre $P$. radiata la aplicación de los fungicidas redujo significativamente el índice de esporulación de B. cinerea (IET 1,07), obteniendo un nivel de control de 77,8\%. Clonostachys A10, también fue capaz de controlar el $($ IET 1,21), aunque el resultado fue inferior al tratamiento con fungicidas. Las cepas de Trichoderma A6 y A27 y Clonostachys A11 no tuvieron efecto en la reducción del patógeno, con una incidencia que fluctuó entre $55,4 \%$ y $71,9 \%$ (cuadro 5).

En relación a la severidad, expresada como proporción del tejido foliar con esporulación de $B$. cinerea, las tendencias fueron semejantes a los resultados de incidencia, sólo detectándose una reducción significativa en la severidad con la aplicación de fungicidas y Clonostachys A10, en relación al control (cuadro 6). Específicamente, se observaron diferencias significativas en el tercio superior de la planta sólo en el caso del fungicida y Clonostachys A10. En el tercio medio, los antagonistas Clonostachys A10 y A11 presentaron diferencias significativas con el control, aunque fueron inferiores al fungicida. En el tercio inferior, todos presentan diferencia significativa con el control y los antagonistas Clonostachys A10 y Trichoderma A27 tuvieron un efecto semejante al fungicida.

Los resultados obtenidos en los ensayos fueron semejantes a los obtenidos en los ensayos de selección in vitro, referente a la efectividad de los antagonistas en la supresión de la esporulación del patógeno, en donde los antagonistas A10 y A11 fueron los más consistentes en el control del patógeno. 
Cuadro 6. Índice de esporulación de $B$. cinerea (SE), en tercio superior, medio e inferior en plantas de $P$. radiata, ensayo vivero Carlos Douglas.

Botrytis cinerea sporulation index (SE), in third up, middle and lower $P$. radiata plants, Carlos Douglas nursery assay.

\begin{tabular}{lcccccc}
\hline \multirow{2}{*}{ Tratamientos } & \multicolumn{2}{c}{ Superior } & \multicolumn{2}{c}{ Medio } & \multicolumn{2}{c}{ Inferior } \\
\cline { 2 - 7 } & IET & Rango & IET & Rango & IET & Rango \\
\hline Switch-Sumisclex & $1,10 \mathrm{a} *$ & $1,07-1,15$ & $1,05 \mathrm{a}$ & $1,02-1,08$ & $1,05 \mathrm{a}$ & $1,05-1,08$ \\
Clonostachys (A10) & $1,40 \mathrm{~b}$ & $1,10-1,72$ & $1,16 \mathrm{~b}$ & $1,05-1,31$ & $1,07 \mathrm{a}$ & $1,00-1,16$ \\
Clonostachys (A11) & $1,54 \mathrm{c}$ & $1,42-1,67$ & $1,17 \mathrm{~b}$ & $1,08-1,33$ & $1,16 \mathrm{~b}$ & $1,02-1,25$ \\
Trichoderma (A27) & $1,69 \mathrm{c}$ & $1,41-1,91$ & $1,30 \mathrm{c}$ & $1,08-1,56$ & $1,11 \mathrm{a}$ & $1,08-1,16$ \\
Trichoderma (A6) & $1,66 \mathrm{c}$ & $1,52-1,88$ & $1,41 \mathrm{c}$ & $1,35-1,45$ & $1,21 \mathrm{~b}$ & $1,11-1,32$ \\
Control (**) & $1,55 \mathrm{c}$ & $1,42-1,83$ & $1,25 \mathrm{c}$ & $1,12-1,40$ & $1,32 \mathrm{c}$ & $1,28-1,38$ \\
\hline
\end{tabular}

* Letras diferentes en la columna indican diferencias significativas $(P<0,05)$.

** Sólo aplicación de B. cinerea.

\section{DISCUSIÓN}

Desde el filoplano de plantas de E. globulus y $P$. radiata en vivero, fue obtenido un total 144 cepas de hongos, de las cuales cerca del $50 \%$ fueron ensayadas contra B. cinerea. De estas el $26,8 \%$ presentó los mayores niveles de control, con esporulación del patógeno menor a $25 \%$ (IET $\leq 2)$, seleccionándose ocho cepas, pertenecientes a los géneros Clonostachys y Trichoder$m a$ (cuadro 2), que incluyen especies ampliamente citadas como antagonistas para el control de B. cinerea (Sanfuentes y Ferreira 1997, Peng y Sutton 1991, Swadling y Jeffries 1995, Elad et al. 1996).

Los hongos potenciales antagonistas fueron aislados desde tallos y hojas de E. globulus y acículas de P. radiata, para aumentar la probabilidad de obtener cepas compatibles con el huésped y de esta forma incrementar la posibilidad de éxito en el biocontrol de $B$. cinerea. En este sentido, se ha planteado que el lugar más apropiado para la obtención de antagonistas es el propio hospedero o del mismo ambiente donde será utilizado (Peng y Sutton 1991, Bettiol 1991). Peng y Sutton (1991), Elad et al. (1996) y Sanfuentes y Ferreira (1997) utilizaron un método semejante en la selección de antagonistas y constataron la existencia de microorganismos en el filoplano de diversas plantas, capaces de competir con B. cinerea, obteniendo elevados niveles de control.

Entre las cepas de hongos que ejercieron control sobre $B$. cinerea (IET $<2$ ), algunas poseen antecedentes de ser patógenos sobre especies vegetales. Cladosporium spp. es un hongo común en el aire y que se ha detectado sobre especies frutales y ornamentales, produciendo manchas foliares, o como hiperparásito sobre tumores ocasionados en las plantas por otros patógenos (Ferreira 1989, Elad 1996, Smith et al. 1992). Pestalotiopsis es conocido como patógeno débil, que afecta a hospederos debilitados, el que ha sido observado en vivero provocando daño sobre
Eucalyptus (Ferreira 1989) y algunas coníferas (Smith et al. 1992). En cuanto a cepas de Penicillium que mostraron eficiencia en el control in vitro contra $B$. cinerea, algunas especies pueden ocasionar importantes problemas en poscosecha, provocando pérdidas por pudrición en frutas y bulbos (Elad et al. 1994, Smith et al. 1992, Sutton y Peng 1993). Debido a que estos hongos son potenciales patógenos en otros cultivos, fueron descartados para los ensayos siguientes, a pesar de los buenos niveles de control alcanzados.

Las cepas antagonistas Trichoderma (A6, A27, A30, A58) y Clonostachys (A10, A11, A14 y A30) fueron capaces de reducir la esporulación de $B$. cinerea, en todos los ensayos de selección in vitro. Los resultados fueron comparables a los obtenidos por Peng y Sutton (1991), Köhl et al. (1994) y Zhang et al. (1994) en ensayos similares, con niveles de supresión del patógeno para cepas de estos géneros entre 53 y $75 \%$, indicando el potencial de los hongos ensayados para reducir la enfermedad en condiciones de campo.

En el ensayo realizado in vitro, que consideró previa aplicación del patógeno ( $24 \mathrm{~h}$ antes), se ratificó la efectividad de los agentes en el control de B. cinerea (cuadro 3). Los antagonistas al reducir la colonización y esporulación del patógeno en estas condiciones permiten predecir que estos hongos poseen una rápida germinación e inician la actividad antagónica oportunamente (Sutton y Peng 1993).

La aplicación de este método in vitro de selección de antagonistas a $B$. cinerea ha proporcionado una confiable indicación de la actividad de supresión de los organismos en condiciones de campo (Peng y Sutton 1991), lo que fue confirmado con los ensayos realizados en viveros forestales en esta investigación.

En el ensayo realizado con E. globulus en vivero, la presencia de Clonostachys y Trichoderma sobre la superficie foliar de las plantas confirma que ambos hongos 
son capaces de permanecer por un tiempo determinado y competir con otros microorganismos habitantes naturales del filoplano, atributo importante para la efectividad de antagonistas para el control de $B$. cinerea. La capacidad de los antagonistas de permanecer viables durante períodos secos, y colonizar el sustrato rápidamente, es característica de un antagonista adaptado al nicho de un tejido necrótico (Köhl et al. 1994, Zhang et al. 1994).

En este ensayo, las cepas de Clonostachys y Trichoderma redujeron la incidencia del patógeno entre 45-90\% y superando la eficiencia del fungicida. Peng y Sutton (1991, 1993) obtuvieron resultados semejantes en ensayos de campo, donde $T$. viride y $C$. rosea fueron capaces de suprimir $B$. cinerea, superando a los fungicidas, alcanzando incluso $100 \%$ de control con la aplicación de C. rosea. Los resultados obtenidos en E. globulus (cuadro 5), referente a la superioridad de los antagonistas con respecto al fungicida, ha sido verificada en otras investigaciones y es considerado una evidencia para un eventual desarrollo comercial del producto. Köhl et al. (1994), aplicando Daconil M (clorothalonil + maneb), detectaron que este fungicida no fue capaz de disminuir la esporulación del patógeno. Swadling y Jeffries (1995), utilizando cepas de Trichoderma sp., fueron tan efectivos como el fungicida diclofluanid, en el control del patógeno en frutillas. Peng y Sutton (1990) citado por Zhang et al. (1996) determinaron que cepas de $C$. rosea y $T$. viride fueron más efectivos que el fungicida Captan en la reducción de la pudrición de tomates y habas, tanto en ensayos de laboratorio como de invernadero.

En $P$. radiata, la cepa de Clonostachys A10 redujo significativamente la incidencia y la severidad del "moho gris", aunque logrando un nivel de control que puede considerarse sólo como moderado (40,3\%). Este resultado, no completamente satisfactorio, puede ser explicado en parte por el efecto de ciertos factores como: ambientes extremadamente favorables para el desarrollo de la enfermedad, alta población del patógeno $\left(10^{5}\right.$ conidias $\left./ \mathrm{ml}\right)$ y, además, de algunos daños en la región apical de las plantas. De esta forma, al aumentar la cantidad del antagonista en relación a la población natural del patógeno, mayor periodicidad de las aplicaciones y determinar el momento oportuno de establecer los antagonistas, es posible aumentar las posibilidades de un control exitoso.

No es posible realizar mayores comparaciones entre los resultados obtenidos en los ensayos de invernadero, ya que existieron diferencias metodológicas entre estos. Sin embargo, el mayor periodo de tiempo comprendido entre la aplicación del patógeno y de los antagonistas $(48$ h), en el caso del ensayo en $P$. radiata, pudiera ser un factor que determinó el menor efecto de los antagonistas en el control de la enfermedad. Es conocido que la germinación de las conidias de $B$. cinerea es muy rápida, bajo condiciones ideales de temperatura y humedad, al igual que la penetración e infección (Peng y Sutton 1991, 1993).
De acuerdo a los dos ensayos de vivero, el control químico, si bien es cierto es una buena alternativa, no fue completamente eficiente, ya que en ambos casos existió una incidencia del patógeno no aceptable para la empresa (cuadros 4 y 5).

En el ensayo en E. globulus, las cepas de Clonostachys y Trichoderma fueron más efectivas que el fungicida aplicado. Este comportamiento no ocurrió en el ensayo en $P$. radiata, donde los fungicidas sistémicos cyprodinil+fludioxonil (Switch) y procymidona (Sumisclex) fueron superiores a los antagonistas. Estos productos son eficientes para controlar B. cinerea durante los procesos de germinación de conidias en la superficie de las hojas, penetración y colonización, pero su efecto es restringido en tejidos necrosados de la planta (Cabral y Cabral 1995). Los resultados variables obtenidos con los fungicidas auguran algunas de las ventajas que presenta el control biológico sobre el químico. Por otro lado, el efecto de los antagonistas sobre B. cinerea puede ser directo, creciendo sobre él, incluso después de la formación de estructuras reproductivas y de protección, inhibiendo la actividad del patógeno, como también estimulando respuestas de resistencia en la planta (Elad et al. 1998).

Los resultados de este estudio demuestran que la aplicación de cepas de antagonistas seleccionadas, especialmente de Clonostachys, tienen efecto en el control de $B$. cinerea, y proporcionaron antecedentes en relación a la relativa capacidad de estas cepas en la supresión del patógeno, tanto in vitro como en los ensayos in vivo.

\section{AGRADECIMIENTOS}

A la empresa Forestal Mininco S.A. por el financiamiento para desarrollar esta investigación y las facilidades otorgadas por personal del vivero "Carlos Douglas". A la empresa Biocaf por facilitar su infraestructura para ensayos.

\section{REFERENCIAS}

Barnes S, M Shaw. 2003. Infection of Commercial Hybrid Primula Seed by Botrytis cinerea and Latent Disease Spread Through the Plants. Phytopathology 93(5):573-578.

Bettiol W. 1991. Seleção de microrganismos antagõnicos a fitopatógenos. In Bettiol W ed. Controle Biológico de Doenças de plantas. Brasilia, Brasil. EMBRAPA-CNPDA. $388 \mathrm{p}$.

Butin H, H Peredo. 1986. Hongos parásitos en coníferas de América del Sur con especial referencia a Chile. BerlinStuttgart, Alemania. Biblioteca Mycológica. 100 p.

Cabral S, J Cabral. 1995. The fungistatic and fungicidal activity of vinclozolin against Botrytis cinerea. Mycological Research 99(9):1041-1046.

Chaves N, A Wang. 2004. Combate del Moho Gris (Botrytis cinerea) de la Fresa mediante Gliocladium roseum. Agronomía Costarricense 28(2):73-85. 
Cole M., T Wiechel, M Warren, R Holmes. 2004. Ensuring Optimal Quality Through Management Strategies For Botrytis cinerea. Department of Primary Industries. Monash University. Australian Government. 66 p.

Coley-Smith J, K Verhoeff, W. Jarvis. 1980. The biology of Botrytis. London, England. Academic Press. 318 p.

Elad Y, B Kirshner, N Yehuda, A Sztejberg. 1998. management of powdery mildew and gray mold of cucumber by Trichoderma harzianum $\mathrm{T} 39$ and Ampelomyces quisqualis. BioControl 43:241-245.

Elad Y, G Zimand. 1991. Experience in integrated chemicalbiological control of grey mould (Botrytis cinerea). Contribution from the Agricultural Research Organization. Agriculture Volcanic Center. Bet Dagan, Israel 3274:195-199.

Elad Y, J Köhl, N Fokkema. 1994. Control of infection and sporulation of Botrytis cinerea on bean and tomato by saprophytic bacteria and fungi. European J. Plant Path. 100:315-336.

Elad Y, N Malanthrakis, A Dik. 1996. Biological control of Botrytis - incited diseases and powdery mildews in greenhouse crops. Crop Protection 15(3):229-240.

Elad Y. 1996. Microbial Suppression of infection by foliar plant pathogens. The European Foundation for Plant Pathology Working Group on Biological Control. Bulletin OILB/SROP 16(11):3-7.

Esterio M, J Auger. 1997. Botrytis: nuevas estrategias de control cultural, biológico y químico en uva de mesa. Santiago, Chile. Universidad de Chile, Facultad de Ciencias Agrarias y Forestales, Departamento de Sanidad Vegetal. $125 \mathrm{p}$.

Ferreira F. 1989. Patología Florestal. Principias Doenças Florestais no Brasil. Viçosa, Brasil. Sociedade de Investigações Florestais. 570 p.

Figueredo A, C García, A Grigoletti. 2001. Doenças do eucalipto no sul do Brasil: identificação e controle. $18 \mathrm{p}$. (Circular Técnica No 45 Embrapa).

Köhl J, W Molhoek, C Van Der Plas, N Fokkema. 1994. Effect of Ulocladium atrum and other Antagonists on Sporula- tion of Botrytis cinerea on Dead Lily leaves Exposed to Field Conditions, DLO Research Institute for plant Protection. Phytopathology 84(4):393-401.

Pande S, G Singh, J Narayana, M Bakr, P Chaurasia, S Joshi, C Johansen, S Singh, J Kumar, M Rahman y C Gowda. 2001. Integrated Management of Botrytis Gray Mold of Chickpea. India: International Crops Research Institute for the Semi-Arid Tropics. $32 \mathrm{p}$.

Peng G, J Sutton. 1991. Evaluation of microorganisms for biocontrol of Botrytis cinerea in Strawberry. Can. J. Plant Path. 13:247-257.

Sanfuentes E, F Ferreira. 1997 Avaliação de fungos para biocontrole de Botrytis cinerea em viveiros suspensos de eucalipto. Revista Árvore 21(1):147-153.

Sharabani D, D Shtienberg, Y Elad, A. Dinoor. 1999. Epidemiology of Botrytis cinerea in Sweet Basil and Implications for Disease Management. Plant Disease. 83(6):554-560.

Smith I, J Dunez, D Phillips, R Lelliott, S Archer. 1992. Manual de Enfermedades de las Plantas. Madrid, España. Ediciones Mundi-Prensa. $671 \mathrm{p}$.

Sutton J, G Peng. 1993. Biocontrol of Botrytis cinerea in strawberry leaves. Phytopathology 83:615-621.

Swadling I, P Jeffries. 1995. Isolation of Microbial Antagonists for Biocontrol Of Grey Mould Disease Of Strawberries. Biocontrol Science and Technology 6:125-136.

Yu H, J Sutton. 1997. Morphological development and interactions of Gliocladium roseum and Botrytis cinerea in raspberry. Can. J. Plant Path. 19:237-246.

Yu H, J Sutton. 1999. Density dynamic of Gliocladium roseum in relation to biological control of Botrytis cinerea in red raspberry. Can. J. Plant Path. 21:23-32.

Zhang P, J Sutton W Tan, A Hopkins. 1996. Gliocladium roseum reduces physiological changes associated with infection of black spruce seedlings by Botrytis cinerea. Can. J. Plant Path. 18:7-13.

Zhang P, J Sutton, A Hopkins. 1994. Evaluation of microorganisms for biocontrol of Botrytis cinerea in container- grown black spruce seedlings. Can. J. For. Res. 24:1312-1316. 